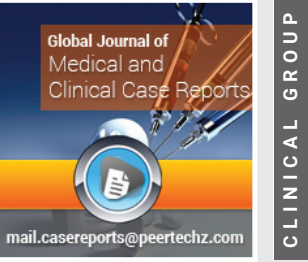

\title{
Metacognitive skills training effect on cognitive function in traumatic brain injury patients:
} A systematic review

Received: 21 October, 2020

Accepted: 02 November, 2020

Published: 03 November, 2020

*Corresponding author: Sara A Makka, Intern Neuropsychologist, Intern Clinical Psychologist, Beirut, Lebanon, Tel: +96176706843;

E-mail: saramakke96@gmail.com

Keywords: Metacognition; TBI; Cognitive outcome; Cognition; Metacognitive skills

https://www.peertechz.com

\author{
Sara A Makka* \\ Intern Neuropsychologist, Intern Clinical Psychologist, Beirut, Lebanon
}

\begin{abstract}
Metacognition is among the current controversial neuropsychological topics that influence perspective of cognition in ill and healthy individuals. Many cognitive failures that Traumatic Brain Injury (TBI) patients endure are not tackled in standardized protocols of cognitive rehabilitation efforts. One of the debilitating features of TBI is the lack of self-awareness of the disease and its corresponding cognitive failures. Cognitive failures can be a result of both cognitive decline due to insult and lack of awareness of error detection that is secondary to the injury. Metacognitive training can help in diminishing rehabilitation resistance obstacle. Not many studies have been done on meta-cognitive rehabilitation efforts in the case of TBI patients. In this review, electronic database is searched in strict inclusion exclusion criteria and 393 studies were identified from two database and grey literature. Eight studies were included in a descriptive analysis after selecting clinical trials which aimed to elevate cognitive components outcome in TBI patients through applying metacognitive training. This systematic review might determine the power of evidence on the effect of metacognitive skills and strategy training on cognitive function in TBI patients regardless if cognitive outcome is measured through a holistic instrument or if the training has aimed to elevate one component or more of cognition. Studies recruited ranged from addressing executive function, social skills, to general cognitive competences. All of the studies proved the significance of metacognitive interventions in altering cognitive rehabilitation outcome as it had been interpreted by the conductors of the trials themselves but more attention should be given to the generalization of learned skills to daily life. Further details regarding the usage and implementation of metacognitive training are discussed and finally metacognition is judged to be an essential component that must be better addressed in cognitive and non-cognitive rehabilitation in TBI patients.
\end{abstract}

\section{Introduction}

TBI can influence several levels of cognitive function depending on the severity and site of the injury. One of the most weakening consequences is the unawareness of the patient's error or the illness itself. This stands out as a great hinderer for rehabilitation efforts. The knowledge of someone's circumstances can be referred to as meta-knowledge. Metacognition is similarly the cognition of cognition or, if put simple, the ability to introspect personal thought and cognition. Metacognitive interventions aim to elevate the patient's awareness of his situation and the surrounding and teach him better strategies to address his fallibilities. The main strength that the injured patient gains with metacognitive training is realizing his errors and increasing his capacity to auto-correct them. If metacognitive interventions proved successful enough to be a part of cognitive rehabilitation protocol, many rehabilitation resistance can be decreased by raising the self-awareness that is the personal awareness of errors and self in the patient's eyes. In addition to raising the efficacy of rehabilitation, many caregivers and family members will find better guided ways to deal with the patient as they better understand the "state of denial" they accuse the patient to produce. Metacognitive training can also address other functional domains of cognition that can be later on investigated. Literature has been conducted on the efficacy of metacognitive training or other approaches which have been formulated based on metacognitive principles and have proved themselves significant. With that mentioned, metacognitive training doesn't seem to be the most spontaneous and direct preliminary step done when conducting psychometric assessment for posthospitalization care but it is still realistically poorly addressed. To better assess where metacognitive training and strategy training had evolved and hence decide whether cognitive 
rehabilitation and TBI assessment protocol classification steps should be reconsidered, evidence should be accumulated and collected and clinicians' awareness of metacognition effect on rehabilitation should be raised. This will help clinicians include metacognitive training as an initial pre/post assessment outcome and a protocol in neuro-rehabilitation and cognitive rehabilitation in TBI patients. In this study, we will focus on highlighting the importance not the strategy itself of metacognitive training in different domains (executive function, social skills, memory, etc...). The direct objective of this conducted systematic review is to possess enough scientific evidence on the importance of metacognitive assessment inclusion in the post-hospitalization procedure of TBI patients in practice and in research.

\section{Literature review}

\section{Setting definitions}

TBI occurs when a force transmitted to the head or body results in neuropathologic damage and dysfunction [1].

Another definition for TBI is provided by Medscape being a non-degenerative and non-congenital insult to the brain from an external mechanical force. In this definition, the term "external" will exclude all internal causalities of brain injury and hence exclude other causalities of brain injury that are not directly resulting from TBI.

TBI definitions are consistent on being an injury to the head. In medical literature, the terms head injury and TBI are used interchangeably.

Centers for Disease Control and Prevention defines TBI as follows;

"A TBI is caused by a bump, blow, or jolt to the head that disrupts the normal function of the brain. Not all blows or jolts to the head result in a TBI."

This means that traumas which cause brain (note that not strictly cognitive but any neuropathologic consequence or any other sort of functioning) malfunctioning will be considered as TBI and hence if a patient simply bumps his head without any significant brain malfunction shall not be considered as a TBI. This disintegrates the usage of the term "head injury" in medical literature as "traumatic brain injury" and places head injuries as a broader set since the difference lies in the neuropathology resulted from the trauma. This is important to be noted out because in our systematic review, we do not include general head injuries but TBI in specific.

Over here, a concern arises regarding whether this classification applies to head traumas; will traumatic brain injuries be solely classified as traumatic brain injuries if the trauma causes neuropathology on onset and not over a period of time and through worsening?

The definition of The US Department of Veterans Affairs and the Department of Defense's Clinical Practice Guideline for Management of Concussion/mild TBI resolves this dilemma as it states the following:

"TBI is a traumatically induced structural injury and/or physiologic disruption of brain function as a result of an external force... etc"

This means that more focus is given on the structural form of the injury previous to the neurologic cascade that it might result in.

Besides this definition, another essential rehabilitation oriented concern arises; "When and how does a clinician classify brain malfunctioning to better diagnose TBI?" The diagnostic criteria of TBI does resolve this controversy as well, by quantifying the severity of the trauma to brain using assessment scales which will be dissected afterwards.

\section{Tracking the term}

It is important to track the term "TBI" before by naming the first steps to its discovery as its own concept evolved. It was mentioned as early as the $14^{\text {th }}$ century when Berengario da Carpi, the Italian anatomist, published a revolutionary paper titled "De Fractura Cranei" or "skull fracture". This monograph was dedicated for head injury and came after Berengario treated a serious head injury in Lorenzo de Medici [2].

Brain injury was even earlier mentioned in 3000-2500 B.C when the pyramids were under construction and it was discovered in the Edwin Smith Papyrus which is an ancient medical Egyptian text. The text discussed 27 head injuries (13 of which were neuropathologically involved) and classified them into three categories depending on the patient's ability to stay independent after the trauma - which is also still relevant to the current assessment in post-injury medical care and rehabilitation processes [2].

Gonzallo Bertullo claims in his publication titled as "History of TBI" that the greatest advances of the knowledge of the neurological function did not come until the nineteenth century. Until then, head injuries were still classified by the skull fracture and not the corresponding neurological examination.

All of this discussion regarding the understanding of terminology does seem linguistic or literal but is actually definitive when it comes to treatment and rehabilitation of TBI. Furthermore, it is especially essential to review definitions as they pave the way for conceptual development, understanding, and also highlight the importance of the classification criteria discussion.

\section{Prevalence and incidence of TBI}

TBI is a leading cause of chronic disability worldwide especially in those below their forties [2]. Studies show that around 775,000 adults live with long-term disability due to TBI. The incidence and prevalence rates of TBI differ among clinical and epidemiological studies. These differences might be due to mainly the variance in the inclusion and exclusion criteria proposed when conducting the study or collecting data, 
the difference in the participants' characteristics as well as in the classification criteria within and across subtypes. This is still, however, a critical problem that is very demanding when it comes to understanding rehabilitation efforts. One of the important factors that affects the statistical count is the method of report chosen to be elected. TBI patients who do not seek medical care might not be included. Therefore, the estimates might drastically change despite of other considerations. 27 million new cases of TBI were reported worldwide in 2016. Even though those numbers mean that around $2.5 \%$ of those who experience a TBI die, it is prevailing that an ample number of patients with TBI do not seek medical care post-injury. This leaves us with less statistics and more disabled humanitarian capital not significant enough for career, integration, research, and other life capacities.

\section{Different classifications of TBI}

TBI can be penetrating and non-penetrating. The nonpenetrating TBI can result from either the direct traumatic event impact or from the rapid head acceleration or deceleration without impact. This mechanism will result in two phases; direct result of traumatic event and a cascade of consequences of neuropathologic processes continuing for days, weeks, or months. Penetrating TBI will cause direct damage to brain tissue since the forcing object will be able to enter the brain (TBI - Symptoms and Causes - Mayo Clinic). It is good to know that blast- induced TBI is becoming more and more common among military head injury. It is caused by the transfer of the shock wave from the blast to the cerebrovascular system or brain tissue and causes neuronal damage [3].

\section{Severity of TBI}

This classification of injury is different from the classification of the severity of TBI and is also distinct from the classification of the disability arising from the injury.

During the diagnostic procedure, a clinician will usually assess the severity of the trauma in a typical manner; early neuroimaging assessing the presence of altered consciousness, post-traumatic amnesia, and applying the Glasgow Coma Scale score. The Glasgow Coma Scale is considered as the gold standard of neurologic assessment and is used to assess coma and impaired consciousness and it is special for having multiindicators, while other grading scores consisted of single indicators [4].

Severity of TBI can range from mild, moderate, to severe. Mild traumatically brain injured patients will experience a slight to no loss/alterations of consciousness. Four factors are used to determine the severity of TBI; neuroimaging results, extent of altered loss of consciousness, length of posttraumatic amnesia, and Glasgow Coma Scale scores. To classify severity more specifically, Table 1 is presenting.

Of course, several other classifications are present and each of the following organizations participated in providing a criterion; World Health Organization, Centers for Disease Prevention and Control, Mayo Classification System, National

\begin{tabular}{|c|c|c|c|}
\hline Table 1: Severity of TBI determination criteria. \\
\hline Criteria & Mild & Moderate & Severe \\
\hline Structural imaging & Normal & Normal or Abnormal & $\begin{array}{c}\text { Normal or } \\
\text { Abnormal }\end{array}$ \\
\hline $\begin{array}{c}\text { Loss of } \\
\text { consciousness }\end{array}$ & $0-30$ minutes & $\begin{array}{c}\text { Between } 30 \text { minutes and } \\
24 \text { hours }\end{array}$ & $\begin{array}{c}\text { Exceeds } 24 \\
\text { hours }\end{array}$ \\
\hline $\begin{array}{c}\text { Alterations of } \\
\text { consciousness }\end{array}$ & $\begin{array}{c}\text { A moment up to } \\
24 \text { hours }\end{array}$ & $\begin{array}{c}\text { More than } 24 \text { hours and relates to other } \\
\text { severity criteria }\end{array}$ \\
\hline $\begin{array}{c}\text { Post traumatic } \\
\text { amnesia }\end{array}$ & $0-1$ days & $\begin{array}{c}\text { Between a day and } 7 \\
\text { days }\end{array}$ & $\begin{array}{c}\text { Exceeding } 7 \\
\text { days }\end{array}$ \\
\hline $\begin{array}{c}\text { Glasgow coma scale } \\
\text { score }\end{array}$ & $13-15$ & $9-12$ & $\begin{array}{c}\text { Less than } 9 \\
\text { days }\end{array}$ \\
\hline
\end{tabular}

Institute of Neurological Disorders and Stroke, Diagnostic and Statistical Manual of Mental Disorders, and many others.

There is also very little data on screening for potential traumatic brain injuries which have been established in the history of patients occurring without the patient's knowledge. Currently, gold standard self-report along with protein measurement precursors are being constructed to affirm similar cases [1].

\section{Consequences of TBI}

Histopathological and behavioral studies have emphasized that the nature of insult of the initial trauma is progressive. This shall involve multiple pathophysiological mechanisms which includes a cascade leading to prolonged motor and cognitive deficits.

The mechanical force protrusion will make the TBI of a primary result of Trauma. These forces directly damage the neurons, axons, dendrites, glia, and blood vessels in a focal, multifocal, or diffuse pattern and initiate a dynamic series of complex cellular, inflammatory, mitochondrial, neurochemical, and metabolic alterations [1].

The severity of the primary injury resulting from trauma can be altered by the usage of protective measures such as the helmet and similar equipment. Yet once the trauma occurs, the immediate neurologic pathology is inevitable and it is usually not alterable. What is alterable, however, is the neuropathologic damage occurring from secondary injury. Secondary events are the cascade of events that occur as a result to the primary brain injury like ischemic and hypoxic damage, cerebral edema, raised intracranial pressure, hydrocephalus, and others. Axons as shown in the Figure 1 [5] in the white matter and due to their neurofilament nature and their viscoelastic nature are more susceptible to acceleration and deceleration injuries. Brain dysfunction is increased by the increased reduction of blood flow or oxygen below the threshold required or by cerebral herniation. Within hours of the trauma, fluid accumulates in the brain tissue producing cerebral edema [1] as presented histopathologically in Figure 2 [5] and this is why it is essential in the pre-hospital management to prevent hypoxia and hypotension for both standing as the most fatal conditions and it should be done as early as possible.

Specific injuries of TBI can have specific and consistent consequences. Also, the severity of the TBI is determined 

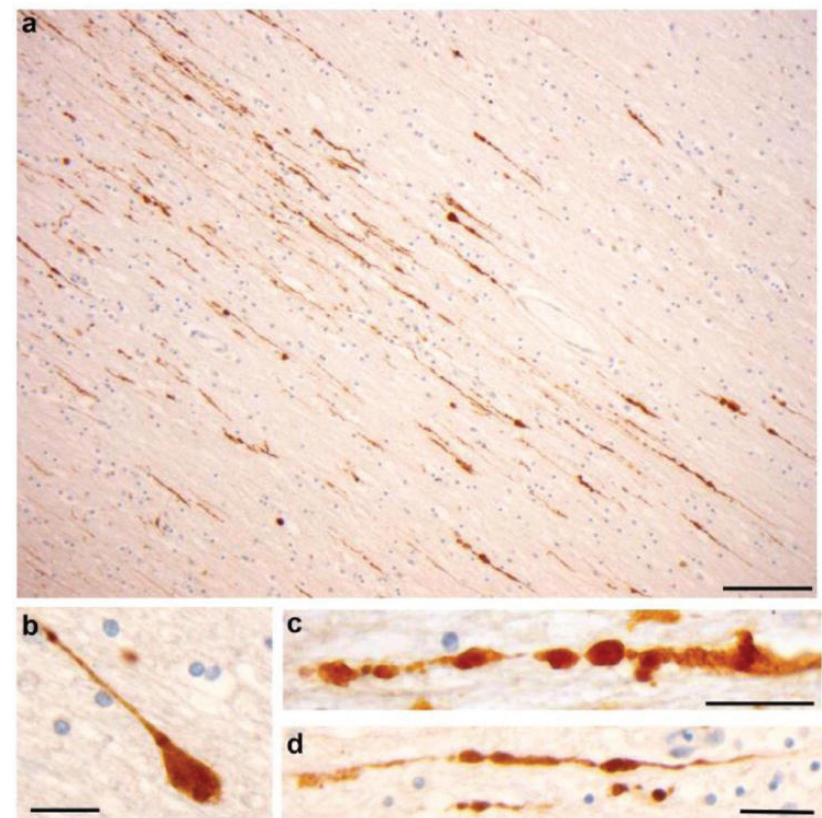

Figure 1: Representative Images of Axonal Pathology Following TBI in Humans Identified Using APP Immunohistochemistry

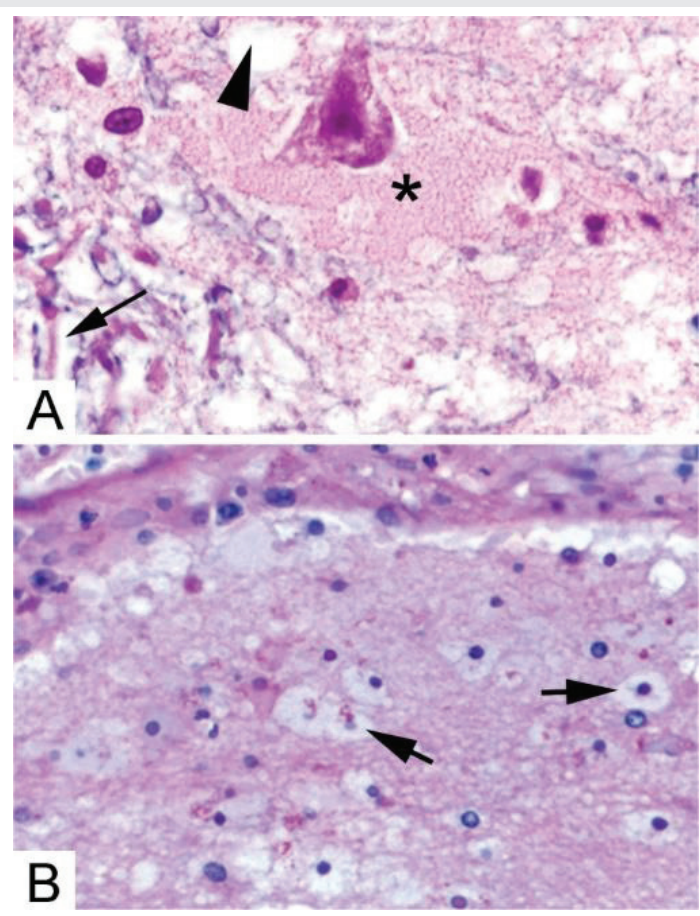

Figure 2: Histologic features of brain edema.

accordingly. Injuries associated with focal pathology have a more varied set of symptoms depending on the anatomy of the injury. When assessing focal injury; Contusions, Skull Fractures, Hemorrhage, and Hematoma are considered. It is more consistent as mentioned above in the case of diffuse injury or in the diffuse aspect of the injury. Brain swelling, vascular injury, excite-toxicity, diffuse axonal injury, and oxidative stress are upon the primary consequences. Mass lesions, such as contusion, subdural hematoma, epidural hematoma, and intraparenchymal hemorrhage are considered focal injuries, whereas diffuse injury encompasses axonal injury, hypoxic- ischemic injury, and micro vascular injury that affect widely distributed anatomic regions [1].

Functional MRI studies support the evidence now that TBI can accelerate the aggregation of certain neurodegenerative proteins or their misfolding and these abnormal brain activation patterns can persist for months after injury including tau, $\beta$-amyloid, $\alpha$-synuclein, and tar DNA binding 43 proteins. A single moderate to severe TBI with loss of consciousness is associated with a two- to fourfold increased risk of dementia in later life. Long term vulnerability to neurodegenerative diseases and brain atrophy is a critical problem when it comes to the management of TBI.

This cascade of events can carry several pathological short and long term consequences, yet we are more concerned about cognitive dysfunctions resulting from the injury. Those insults can be specific to each patient and injury characteristics.

\section{Cognitive consequences of TBI}

Cognitive performance is typically conceptualized in terms of domains of functioning [6]. These domains are hierarchical in nature, with the "lower" referring to more basic sensory and perceptual processes and the "higher" referring to elements of executive functioning and cognitive control. Higher cognitive functions (executive function) are predicated to lower cognitive functions (attention). There are several ways to conceptualize cognitive ability domains. These include classification by the general process involved, such as memory, attention, visuospatial perception, language, and executive function.

At least 85,000 of TBI patients experience and develop cognitive, emotional, behavioral, and somatic disability. Neuropsychiatric sequelae are a broad set of problems including personality changes, aggression, mood disorders, psychosis, postconcussive symptoms, and many other issues that the patients endure producing significant morbidity issues. Cognitive impairment is also a debilitating feature and a substantial source of morbidity as well [7].

Cognitive basic functions are mostly a substrate of the integrated neural network in the frontal subcortical circuits which represents the anatomic and functional extension of those cognitive basics. In this review, we are specifically interested in attention and executive functions.

Attentional processes are cognitively basic and they sprout from different neural networks migrating from the central nervous system which includes the thalamus, reticular formation, hippocampal and entorhinal cortex areas. A disruption in any of the compromising areas can cause a set of clinical symptoms such as coma [7].

Executive function and other complex cognitive functions such as social intelligence and motivation (which circuit is presented in Figure [3] can also be impaired. Comportment that refers to the ability to use self-assessment for integrating in appropriate social behavior, is one of those complex procedures. Lateral orbitofrontal subcortical circuit is mainly ascribed for this process as it integrates limbic and paralimbic 
How Motivation Systems Develop

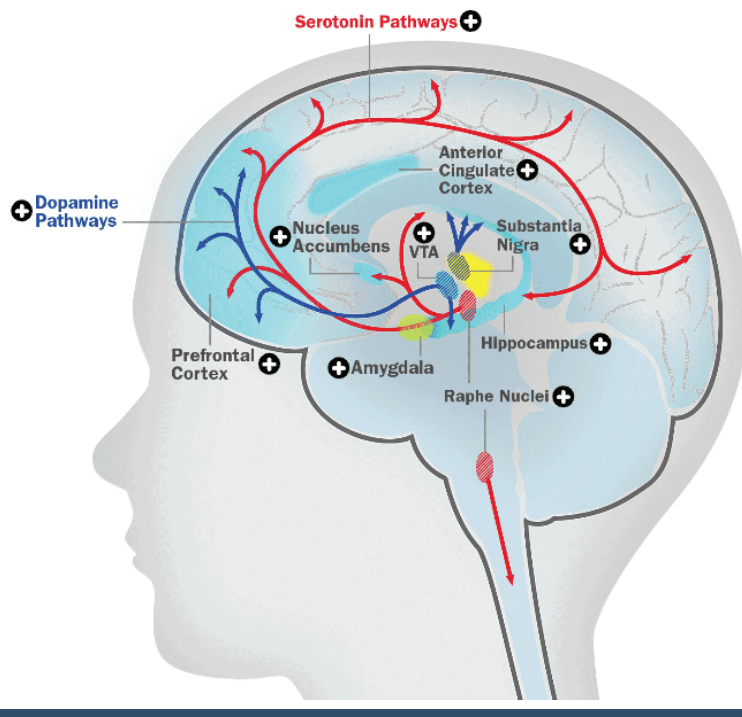

Figure 3: The Brain Circuits Underlying Motivation

information along with the social cues to produce a successful social behavior. The insult to those areas clinically present as aggression for example but not as cognitive decline as reported by the care giver yet it is still considered as an early sign of decline [7].

Disorganization as a result of executive dysfunction is the most common complaint in individuals with TBI [8].

Unawareness of illness can be as well one of the most debilitating features of un-awareness due to the loss of the functional state of this knowledge (due to TBI) for the patient himself and also the caregiver [9].

Even though insult of a penetrating injury to those areas is understandable, other non-penetrating traumas are still difficult to elucidate. Pharmacotherapy is able to treat clinically and symptomatically similar events with multiple etiology which can result in different cognitive improvement and side effects (B.Arciniegas et al, 2002). In other words, pharmacotherapy might treat similar symptoms of different etiology medically speaking unlike psychotherapy and rehabilitation which are more interested in etiology even when the sole goal is therapy.

If we could enhance our neuroimaging instruments and their reading significance which means a better understanding of brain injury and neural circuits, we might thus be able to better tackle the etiology and enhance our rehabilitation strategy. This is not solely achieved by neuroimaging and insult severity assessment criteria but also a result of better understanding of aggregated scientific literature on metacognition and TBI.

\section{Neurorehabilitation in TBI}

Neurorehabilitation in TBI patients ranges from the early rehabilitation starting loss of consciousness until posthospitalization care which includes reintegration of the disabled patients into their social and professional environment (Obelholzer, 2019).
Lesion diversity leads to different clinical patterns which will lead to different pharmacotherapy and also different rehabilitation efforts and planning. Currently, there are no international guidelines for the rehabilitation efforts of TBI patients (Obelholzer, 2019) regardless of the proposed pharmacological therapy. Noninvasive brain stimulation, Hyperbaric oxygen therapy, limb/organ function reconstruction through functional electrical stimulation, and many other rehabilitation techniques are currently present despite the complexity of TBI being a main hinderer in the clinical trials conducted on rehabilitation efforts in this field [2].

Specified cognitive impairments rehabilitation strategies initiate from the neuropsychiatric assessment done for the patient [10] which brings us to an important problem regarding rehabilitation strategies in metacognition. Rehabilitation strategies solely depend on subjective instruments that are limiting making the assessment vulnerable to report bias [11]. Attention, Memory, Language, Visuospatial perception, and Executive function are all goals with variable importance when setting a cognitive rehabilitation plan for a patient yet choosing to undergo a holistic approach that addresses all neuropsychological level usually shows better improvement [10].

Attention process training and tasks for attention deficits, compensatory strategies and errorless learning training for memory deficits, pragmatic language skills and social behavior guidance for cognitive-communication disorder, metacognitive strategy, and problem-solving training for executive disorder are the mainstay of therapy for cognitive deficits in persons with TBI [10].

\section{Metacognition and TBI}

The ability to engage in self-reflective processes is disrupted after neurological compromise as TBI patients show reduced self-awareness as reported by their care givers and clinicians especially to their functional capacities [10].

Metacognition is the cognition of cognition, knowing of knowing, the awareness of being aware, and it is practically the thought of thinking as in the thought of thought. It can take many forms such as the knowledge of how and when to use specific strategies towards problem solving. John.H Flavel, a cognitive psychologist, gave higher cognitive functions the term metacognition meaning literally "above cognition". Metacognition, defined as the ability of an individual to evaluate his or her own cognitive processes, is commonly disrupted after TBI.

Lack of awareness of errors might be one of the issues that has to be tackled in future research in the field of rehabilitation and resistance. Impaired error processing does not support adequate neural feedback to process correction of errors which might carry on ample new inappropriate conditioned reflexes for TBI patients. This impaired awareness is now being supported by electrophysiological and neuropsychological studies that highlight online metacognitive processes accumulate evidence of erroneous responses in a graded fashion [13]. 
Very few studies aiming to improve metacognitive skills such as self-awareness and self-regulation have been reported in TBI [14]. In a study conducted to measure the relevance of gray matter of prefrontal cortex (as metacognition in some literature is considered to be upon the "higher" cognitive functions mentioned above that is executive function which is also linked to the same brain area) and metacognition in TBI, there was a significant difference between studied groups in the prefrontal gray matter volume and metacognition in comparison to control group of TBI. This means that metacognition even when directly or indirectly assessed, is an existing neural circuit or function and not still perceived as a pseudo-science topic.

\section{Metacognitive skills training (MST)}

In this review, metacognition was studied as partly applied, whole applied, or as an assisting technique in rehabilitation processes that were conducted on TBI patients. So cognitive decline is not the aim to be explained especially that it is multifactorial and symptomatic between patients, but the contribution of metacognition to the neuro-rehabilitation of cognitive decline is the aim.

MST is one of the methods used in the cognitive rehabilitation of TBI patients where the approach aims to accelerate the development of self-awareness in patients with TBI. Its main objective is to teach patients to self-monitor their performance. The approach includes self-awareness training, psycho-education, role modeling of strategy use, and also uses verbal, video, and experiential feedback in therapy activities [11].

A rising interest in research had lately reported metacognitive strategy training (directed at improving selfmonitoring and self-regulation) as being more effective compared to conventional rehabilitation in improving posttraumatic executive dysfunction. Metacognitive strategy training helps reduce or prevents errors by structured and repetitive cueing, or by encouraging repeated assessment and self-monitoring. This is better facilitated when complex tasks can be broken into smaller steps and directly teaching individuals using step-by-step procedures [10].

It is now shown that MST facilitates the treatment of attention, memory, language deficits, and social skills [10].

\section{Importance of review}

With the very scarce literature we have on the improvement that MST can produce, whether metacognitive skills are considered a part of executive higher functional level of cognition or a cognitive entity on its own, one must reconsider the importance of MST in the rehabilitation efforts of executive function. A study found significant correlation between some components of executive system and metacognitive selfawareness. This confirms that metacognitive skills such as selfawareness must be addressed contextually in rehabilitation of cognitive function since it influences cognitive decline speed and domains. Many other studies confirm correlation between heightened metacognitive awareness and executive function impairments post-TBI in severe cases in the sense that higher impairments in executive function are associated with lower metacognitive awareness.

In this review, MST is reviewed as an intervention affecting cognitive outcome in TBI patients.

\section{Objective}

This review aimed to determine the effect of MST on cognitive function specific domains or globally on all its domains in TBI patients.

\section{Methods}

Using the "Preferred Reporting Items for Systematic Reviews and Meta-Analyses" (PRISMA) 2009 guidelines, one researcher (myself) independently performed the search using two database namely; National Library of Medicine (Pubmed) and Cochrane Library.

As for mesh terms and term harvesting, keywords and combinations related to "MST", "Cognitive Function", and "TBI" were speculated. The keywords were combined using the Boolean operator (OR and AND). Bibliography lists from all eligible articles were also hand-searched in order to identify additional papers that might be relevant for the topic and to be included. Google Scholar was also reviewed for other potential inclusions and grey literature. Automated limits were excluded as much as possible due to scarce data.

Due to the little data presence, the citation management was achieved without a software and deduplication was asserted by eye.

All articles were retrieved through database search since January 2000 until September 2020 with the term "metacognition" being introduced to research as early as the date mentioned.

All articles were inserted into Mendeley referencing program that is a desktop and web referencing manager for academic scholars.

\section{Criteria for considering studies for this review}

Types of studies: Randomized Clinical Trials were included in addition to single and series case studies which had an experimental design.

Types of participants: Pediatric and adult participants suffering from TBI (mild, moderate, to severe) were recruited. Studies were selected where participants were matched. Participants with head injuries that were not considered as TBI were excluded.

\section{Types of interventions}

Metacognitive intervention as an intervention: Interventions that are specifically targeted at restoring components of metacognition or any of its relevant aspect 
such as self-awareness or mediated learning were included. In this sense, if MST was performed in combination with another technique that would be by definition also metacognitive, the study would be included. However, if the metacognitive intervention was used in combination with another intervention that was not encompassed under metacognition then the study wouldn't be recruited.

Metacognitive intervention as a comparison control: Metacognitive interventions in the systematic review performed in the present study intend to study the effect of MST as the direct cognition effector, however many studies identified used MST as a control rather than an intervention. Similar studies were not included for the review.

An intervention with Metacognitive objectives: It must be noted that some metacognitive interventions would not be in abstract or keywords referred to as "metacognitive" intervention but as "mediated learning" or "error awareness" or any other naming but will be each representing a module within metacognitive components of intervention and will be asserted in the full text of study as a metacognitive intervention.

Types of Outcome Measures: Global cognitive function or components within cognitive function

The gold standard of outcome measurement is pre versus post assessment differential in functional status [15].

The primary outcome is global cognitive function, and the secondary outcomes are components of cognitive function For this cause, any component of cognition or component of cognitive compartments (ex; social competences, executive function, error detection, discourse and speech, etc...) was included.

\section{Eligibility criteria}

- Exclusion criteria:Any literature that is not in English.

- Epidemiological studies.

- Literature published after August 2020 (to receive the most recent updates as some studies were still uploading their results).

- Literature that does not include keywords or their synonyms.

- Studies which combine MST with other cognitive or any kind of interventions.

- Study protocols and reviews were also excluded.

- Trials which had a combination therapy including a pharmaceutical drug directly or indirectly influencing our variables.

\section{Inclusion criteria}

- Trials and experiments and case studies which aim to study the effect of the intervention.
- Studies from January 2000 until September 2020 were included.

- Studies which address pediatric TBI.

- Studies which address cognitive function as a whole or as part (executive function, visuospatial, etc...).

- Pilot studies of MST interventions effect on cognitive decline in TBI were included.

- Case series or single case studies were included if they had an experimental design.

\section{Data collection and analysis}

Selection of studies: The titles of the records obtained from the electronic searches were screened and eliminated when clearly not relevant.

Studies were independently screened from the titles and abstracts of the remaining studies and excluded when deemed irrelevant. Full text of the remaining potentially relevant studies were independently selected eligible for inclusion based on the inclusion criteria already described. When data seemed insufficient, the study was excluded due to the lack of enough time to contact the researcher.

Data extraction and management: A predesigned data extraction form (used from Cochrane guides) was used to extract data from the studies that met the inclusion criteria The form was modified depending on the needs for the review. After various search strategies were computed and results kept on showing as duplicating, the search strategy generation seized. Studies' data were afterwards extracted.

\section{Results}

\section{Electronic search results}

The electronic searches yielded 393 records and--after the initial screening of titles and abstracts, (376) irrelevant papers were eliminated. (21) studies were to be included according to the pre-set inclusion criteria but (11) were excluded for a total of (8) studies to be finally eligible for the descriptive analysis.

Reasons for exclusion were relative to each study aside from the exclusion criteria which were already taken into consideration during identification of studies step. Some of those reasons ranged from; using MST as a control intervention, lack of data on results, having an outcome not clearly representative of cognitive components, etc... Judgment on similar argument has been resolved by resorting to a professional PSY.D (Doctor of Psychology who is legally able to carry out psychotherapeutic work and focuses on the clinical and applied aspects of psychology). Table of excluded studies can be found in addition to the table of included studies in the text of the study in Tables.

Thus, 8 studies were eligible for inclusion (71 participants) in this review $[8,12,14,16-20]$. 

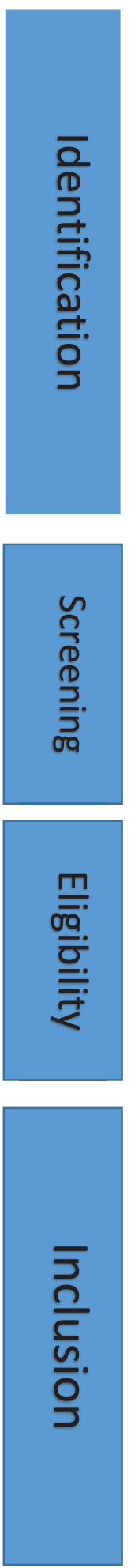

(393) Studies were identified by database search and grey literature

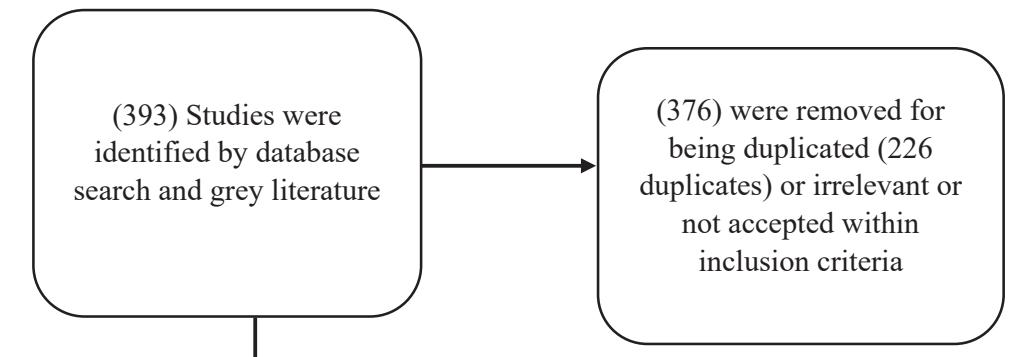
eligible for data extraction, bias assessment, and then metaanalysis

(21) studies were considered for inclusion after being screened twice by same author and assessed for full text

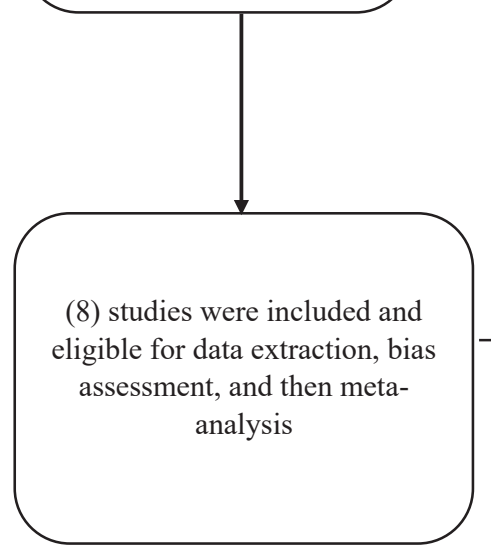

(376) were removed for duplicated (226 not accepted within inclusion criteria

\section{Assessment of bias}

Bias was assessed independently. All included trials for potential sources of bias including selection bias, and performance bias were assessed. The following grading domains (as 'low risk', 'high risk' or 'moderate risk') of bias for each included study was used and documented these grading within the 'Risk of bias' as it is shown below in Table 2. It must be noted that any bias collecting below 2 is considered low, any bias pointing above 7 is considered high risk of bias. Quality assessment checklist for prevalence studies (adapted from Hoy et al. [1]) as it is shown in Table 2. 

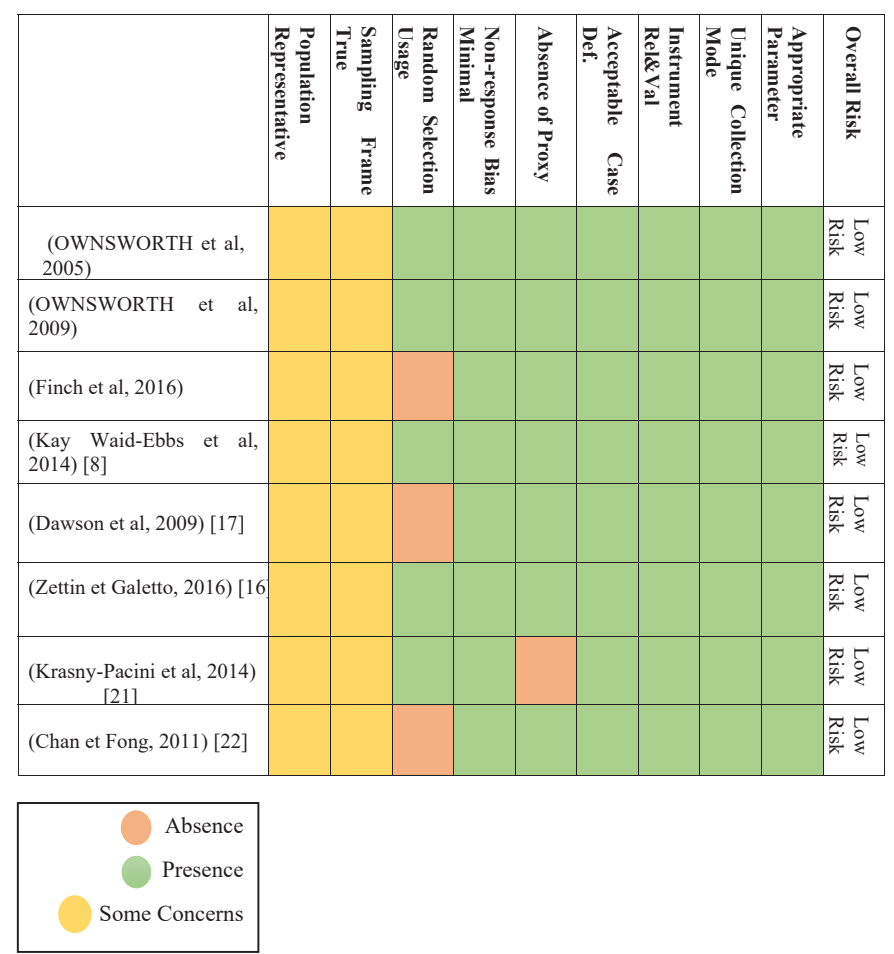

Table 2: Assessment of Risk.

\section{Data synthesis}

Data which was extracted is selected from included studies is presented in table (3) and excluded studies disregarded is presented in Tables 3,4.

\section{Discussion}

\section{Summary of main results}

Out of the 8 studies included, studieswere classified according to their main key of comparison in the field of cognition: Metacognitive interventions targeting error awareness and self-correction $[14,20]$, Metacognitive interventions targeting executive function $[8,17,21]$, Metacognitive interventions targeting social competences [12,16], and Metacognitive interventions targeting global cognitive function with focus on problem solving in one study [22]. All studies proved efficacy and significance of their proposed interventions as the results of their studies have been statistically shown and proved.

\section{Significance of studies}

Metacognitive interventions targeting error awareness and self-correction: It is good to note that executive functioning encompasses both error detection and self-regulation. Interventions which target self-regulation can be considered in the same set ( [23] yet and after reviewing literature and due to the specificity of self-awareness and regulation debilitation as a major concern in TBI, it has been reconsidered as a category on its own within the classification. It is good to mention that studies targeting executive function addressed the executive function as a global function and have not addressed its sub branches. The two studies $([14,20]$ were conducted by the same first author and aimed to apply MST sessions in comparison to other behavioral interventions and used appropriate instruments to measure outcomes and monitor psychological functioning alongside. Both studies showed significant decrease in error frequency and a significant increase in error detection which shows that MST can have a positive effect on error awareness in TBI patients. To better appreciate the importance of MST in error awareness, one must know that poor awareness of behavior, emotions, and disability had been linked to less rehabilitation efficacy and more resistance. Better awareness of mistakes and errors would practically decrease forgetting daily chores (ex: to lock the doors, close the stove, shutdown the lights, and etc...) which will by product increase the patient's independence and decrease the need and worry of the caregiver to stay around [29]. Gaining better cognitive control of consciousness will help the patient enhance his sustained attention versus distractibility that he usually suffers from [30].

Metacognitive interventions targeting executive function: A successful holistic rehabilitation program aims to address the ability to generalize skills remediated at the center to the daily life of the patient. This ability to generalize and extend the mediated learning of skills is better enhanced by cognitive flexibility that is one of the components of executive function $[11,28]$. In those studies, generalization was addressed. Group analysis showed a significant improvement in executive function and the investigation of the efficacy of the Cognitive Orientation to daily Occupational Performance (COOP) approach also showed significant results for the extension of the learned skills to the daily life activities $[8,17,19]$. The third study though used Goal Management Training but showed union of intersection with Metacognitive Skills Intervention and had also extended its area of investigation to detect whether those generalized concepts could extend to long time after the injury [19]. Unfortunately, this was not achieved and the self-informant questionnaires showed that the skills couldn't be generalized from the clinic/center to daily life activities. The study itself recommended further improvements and elaborations on this level even though Goal Management Training proved itself efficacious in the rehabilitation of combat veterans specifically in the executive function aspect. Transfer and generalization of strategy are essential in any executive function enhancement regime [31] because it is not enough to regain skills strictly in clinic. Add to that, our perception and view of learning shouldn't be solely depending on the learned subject. If a clinician is trying to address a certain skill (cooking a meal for example), planning as conveyed by neural behavior will not only elevate the "planning" skill but will generalize to other cognitive compartments since cognitive skills are for classification purpose distinguished but not that independent on a neural level. If generalization fails to be significant then the intervention might (and might not be) successful in clinical contexts only - one of the problems caregivers and family members argue about- because it is of major clinical importance to maintain treatment (Kennedy et al., n.d.). Clinicians are not only interested in documenting impairment but are also interested in developing functional goals of therapy. 
Table 3: Table of Included Studies.
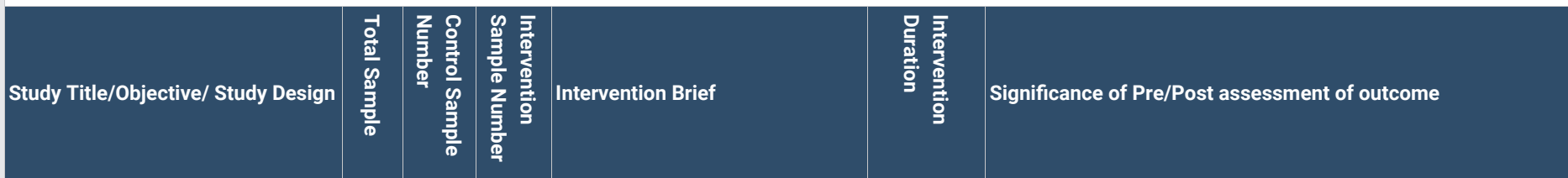

Title: A metacognitive contextual intervention to enhance error awareness and functional outcome following traumatic brain injury

Objective: the present study aimed to investigate neurocognitive psychological, and socioenvironmental factors underlying unawareness for an individual with severe awareness deficits and develop an associated treatment rationale

Study Design: A single-case experimental design

Title:

Error self-regulation following traumatic brain injury

Objective: to evaluate the effects of metacognitive skills training (MST) and behavioral practice on error self-regulation during a naturalistic task after traumatic brain injury (TBI).

Study Design: A case series experimental design

\section{Title:}

Remediation of social

communication

impairments following traumatic

brain injury

using metacognitive strategy

intervention

\section{Objective:}

To perform a pilot study to evaluate whether a novel metacognitive, goal-based intervention improved and maintained the social communication skills of adults with traumatic brain injury (TBI)

Study Design: a pilot

Study of case series experimental design
A metacognitive contextual intervention based on a conceptualization of Neuro-cognitive, psychological, and socio-environmental factors contributing to his (patient) awareness deficits. Intervention targeted error awareness and self-correction in two real life settings: (a) cooking at home; and (b) Volunteer work.

In the first study, after four-session baseline of behavioral practice, two participants received eight MST (Metacognitive Skills Training) sessions followed by four maintenance sessions.

In the second study, a third participant received 16 sessions of behavioral practice to assess the extent to which error selfregulation improves through long term task practice and therapist corrections.

Participants prepared two different meals with a novel meal introduced later to examine skills generalization

Participants with TBI completed three study phases: (1) baseline (2) eight-week intervention targeting social communication impairments and (3) follow-up. The intervention focused on remediating impaired social communication skills using metacognitive strategy intervention and goal-based therapy.

Participants did not receive any intervention (either as part of the study or from other speech pathology services) during the baseline or follow-up phases.
Outcome measures:

Measures of psychological functioning:

1- Marlowe-Crowne Social Desirability Scale (M-CSDS)

2- Symptom Expectancy Checklist (SEC)

Measures of executive functioning:

1- Health and Safety subtest of the Independent Living Scales

2- Tinker Toy Test

3- Five-Point Test

16 weeks 4- FAS test

5- Key Search Test

6- Zoo Map Test from the Behavioral

Assessment of Dysexecutive Syndrome

\section{Significance:}

Relative to baseline performance in the cooking setting, patient demonstrated a $44 \%$ reduction in error frequency and increased self-correction and specific training in this environment led to a $39 \%$ decrease in errors.

\section{Outcome Measure:}

Behavioral outcomes:

1- error frequency checking

2- self-corrections

MST outcomes:

1- Patient Competency Rating Scale (PCRS)

\section{Error behavior:}

A behavioral observation procedure was utilized for assessing error behavior, based on an approach developed in previous research (Hart et al., 1998; Ownsworth et al., 2006).

\section{Significance:}

In the MST study, the two participants demonstrated a $38 \%$ and $76 \%$ reduction in error frequency a significant decrease in checks and a significant increase in self-corrections relative to baseline.

In the behavioral practice study, the participant demonstrated reduced errors (25\%), although this was not statistically significant, and a significant increase in checks but selfcorrections did not significantly change.

This exploratory research suggests that, firstly, by targeting error self-regulation, MST can potentially promote independence on complex everyday tasks.

Outcome Measures:

1- $\quad$ Profile of Pragmatic Impairment in Communication (PPIC)

Two one 2- LaTrobe Communication Questionnaire (LCQ) hour 3- Goal Attainment Scaling (GAS)

therapy session (one individual one group) per week for 8 weeks

\section{Significance:}

Variable changes in PPIC feature-summary scores were observed post-intervention. A non-significant improvement in LCQ scores was also observed. There was a significant increase in GAS goal T-scores following the intervention, with six of the eight participants achieving or exceeding their expected level of performance on all goals. 


\section{Title:}

Response to Goal Management Training in Veterans with blastrelated

mild traumatic brain injury

\section{Objective:}

to test the effect of GMT on

Veterans with executive function deficits following repeated blastrelated $\mathrm{mTBI}$

Study Design:

Controlled trial

Title:

Using the Cognitive Orientation to Occupational Performance (CO-OP) with adults with executive dysfunction following traumatic brain injury

\section{Objective:}

This pilot study tested the applicability of the Cognitive Orientation to Occupational Performance (CO-OP) approach for use with adults with executive dysfunction arising from traumatic brain injury (TBI).

Study Design: case series experimental design

Title:

Efficacy of The Metacognitive

Training on Brain Injured Subjects

\section{Objective:}

$\begin{array}{llll}\text { To assess the effectiveness of such } & 13 & 0 & 13\end{array}$ training I improving metacognitive capabilities and social competences in a group of subject TBI

Study Design: Experimental Design
Goal Management Training

(GMT),

which is a metacognitive intervention, offers an executive function rehabilitation approach that draws upon theories concerning goal processing and sustained attention. GMT is an interactive psychoeducational intervention using seven PowerPoint (Microsoft; Redmond, Washington) modules for guided presentation of information, group interaction, and practice of complex tasks.
The intervention entailed guiding participants to use a metacognitive problem-solving strategy to perform self-identified daily tasks sessions that they needed and wanted to do and with which they were having difficulties.

\author{
Outcome Measure: \\ Measures of Executive Function: \\ 1- $\quad$ Computerized Tower of London (TOL) \\ Generalization Probe: \\ 1- Behavior Rating Inventory of Executive Functions- \\ Adult Version (BRIEF-A)
}

Twice a Significance:

week for 10 Group analysis showed a significant improvement in measures weeks of executive function.

There were no significant changes on self/informant questionnaires indicating a lack of generalization of improvement from the clinic to everyday activities. Overall, while the data indicate efficacy of GMT in the rehabilitation of combat Veterans with executive function deficits because of blast-related mild TBI, enhancement of generalization is needed.

Outcome Measure:

1- The Canadian Occupational Performance Measures (COPM)

2

Dysexecutive Questionnaire (DEX)

20 one-hour Significance: Performance improved to criterion (2-point positive change) on 7 of 9 trained goals and on 4 of 7 untrained goals (self-report). Improvement was maintained at a 3-month follow-up assessment.

The CO-OP approach has the potential to improve performance in daily functioning for adults with executive dysfunction following TBI.

The approach in this field comes from Moritz and colleagues who introduced the Metacognitive Training, a group intervention aimed at enhancing and improving metacognitive abilities in psychotic subjects.

This program is mainly focused on bringing patients' attention on the dysfunctional way in which they integrate social (e.g. expression and gaze direction) or environmental cues in order to evaluate how to behave or to interpret the actions of others. Through a series of drill and practice activities, they are invited to critically reflect about their cognitive biases and to transfer this knowledge in their daily life.

This treatment is divided into eight modules, targeting cognitive errors and problem solving biases which can cause misleading interpretations of the others' mental states and feelings and, therefore, metacognitive difficulties.

\section{Outcome Measure:}

Metacognitive abilities measures:

1- $\quad$ Theory of Mind Assessment Scale (ThOMAS) Social Skills Measures:

Twice a 1- Social Competency Inventory (SCl)

week for 3

months Significance:

As results pointed out, the ThOMAS mean score significantly increased in the second evaluation on the other hand, no significant improvement was found in the SCl between the first and the second assessment

Citation: Makka SA (2020) Metacognitive skills training effect on cognitive function in traumatic brain injury patients: A systematic review. Glob J Medical Clin Case Rep 7(2): 085-099. DOI: https://dx.doi.org/10.17352/2455-5282.000107 
Title: Context-Sensitive Goal

Management Training for Everyday

Executive Dysfunction in Children

After Severe Traumatic Brain Injury

\section{Objective:}

To assess the effectiveness

of a metacognitive training

intervention, based on an adapted

Goal Management Training

and Ylvisaker's principles, on 3

activity domains of executive

functions: (1) prospective memory

(PM) performance in ecological

setting, (2) complex cooking

task management, and (3) daily

executive functioning (EF)

at home and at school

\section{Study Design:}

Single-case experimental

Title: The effects of problemsolving skills training based on metacognitive principles for children with acquired brain injury attending mainstream schools

\section{Objective:}

To investigate the effects of an explicit problem-solving skills training programme based on metacognitive principles for children with acquired brain injury (ABI) who attend mainstream schools.

Study Design:

a controlled clinical trial
The intervention comprised (1) GMT theoretical modules and between-session "missions" (promoting GMT use at home and For 4-6 at school); (2) practical modules months in which children practiced GMT content in meaningful activities: and (3) an "everyday people cognitive coaching guide."

basis

\section{Outcome Measure:}

Executive Function Measure:

1- Children's Cooking Task

Generalization Measure:

1- parental and teacher questionnaires and Goal

Attainment Scaling

Progress Measure:

1- weekly ecological PM score

\section{on a weekly Significance:}

All children improved both on the measure of PM and on questionnaires of daily EF.

Two children improved on the Children's Cooking Task but returned to their pre-intervention level in a novel cooking task at follow-up.

It is feasible but challenging to use Goal Management Training in children with traumatic brain injury.

The participants in the experimental group received problem-solving skills training based on metacognitive principles, while those in the comparison group were on a waiting list to receive the experimental intervention shortly after the intervention in the experimental group had been completed. All participants were measured pre- and post-intervention using measures of abstract reasoning metacognition, problem-solving functional behaviour in the home environment or social situations and individual goal-directed behavior.

The control condition in the comparison group at the time of the experimental intervention received usual care - no active rehabilitation except from attending school classes. An adult training programme was modified for the experimental group to suit the needs of children. This metacognitive training approach was based on the classification of metacomponents by Clements and Nastasi.

\section{Outcome Measure:}

1- Test of Nonverbal Intelligence-3 (TONI-3) which measured non-verbal abstract reasoning abilities

\section{2- $\quad$ Metacomponential Interview (MI) which measured} metacognition

\section{3- Interpersonal Negotiation Strategies Interview} (INSI) which measured problem-solving functional behaviour in social situations

4- Behavior Rating Inventory of Executive Function sessions (BRIEF) which measured problem-solving functional behaviour in the home environment

with each 5- Canadian Occupational Performance Measure with each (COPM) which measured self-perceived goal-directed one lasting functional behaviour individually

for $3 \mathrm{~h}$,

over a

7-week

period.

Significance: Significant differences in post-test scores were found for all measurements between children in the experimental

group and those in the comparison group, using the baselines of dependent variables, years of schooling and the full IQ scores as the covariates.

The results of this study supported the use of explicit problem solving skills training to improve daily functioning for children with $\mathrm{ABI}$, and the need for a larger-scale, randomized controlled study with long-term

follow-up

\section{Metacognitive interventions targeting social competences:} Social communication was addressed in two studies $[12,16]$. Yet, in the Remediation of Social Communication Study, the metacognitive intervention targeted social communication but the outcome of social communication was assessed by linguistic measures which divert the focus from a social context to a language discourse one (Profile of Pragmatic Impairment in Communication (PPIC) was used in addition to other instruments). Nonetheless, discourse is an essential component in social skills and communication especially that cognitive functional domains include language and cannot be perceived as unique circuits each serving the function on its own [32]. The second study was a modified intervention that was originally created to rehabilitate schizophrenic patients and had been divided to eight interesting modules that do not solely focus on social skills or communication [16]. All studies showed significant results. It is good to mention that even though social communication is a very prominent feature in many brain injury conditions as well as neuropsychiatric conditions, up until 2019 there has not been gold standard social competencies assessment tools [33-35]. This means that the significance of the upper mentioned interventions can be 


\section{Study Title}

Connecting Self-Awareness and Error-Awareness in Patients with TBI [13]

Discourse-based treatment in mild TBI [23]

Ready! Set? Let's Train!: Feasibility of an intensive attention training program and its beneficial effect after childhood TBI [24]

Self-awareness assessment during cognitive rehabilitation in children

with acquired brain injury: a feasibility study and proposed model of child anosognosia [25]

Significance of the Feuerstein approach

in neurocognitive rehabilitation [26]

A preliminary report on the effect of

cognitive rehabilitation therapy in

improving cognitive function of

attention following mild traumatic

brain injury: A randomised controlled trial [27]

Cognitive Rehabilitation: ACTION Training for Soldiers With Executive Dysfunction (ACTION)

Neural Bases of Cognitive Rehabilitation for Brain Injury

The Effect of Cognitive Rehabilitation Therapy in Improving Cognitive Function of Attention Following Mild TBI

Cognitive Remediation in pediatric TBI () [18]

Long-term Benefits of an Early Online Problem-solving Intervention for Executive Dysfunction After TBI in Children [19]
Reason for Exclusion

Intervention not proved metacognitive

Intervention not proved metacognitive

No results were provided

Intervention not proved metacognitive

Intervention not proved metacognitive

No results were provided

No results were provided

No results were provided

Intervention not proved metacognitive

No results were provided

No results were provided enhanced by the reliability and sensitivity of the instrument used.

Metacognitive intervention targeting global cognitive function with focus on problem solving: In this single study, problem solving principles depending on metacognitive concepts were trained and administered and were to be investigated without a prior direction or direct outcome measure. For this cause and as it can be seen, 5 outcome measures were selected and used; Test of Nonverbal Intelligence-3 (TONI-3) which measured non-verbal abstract reasoning abilities, Metacomponential Interview (MI) which measured metacognition, Interpersonal Negotiation Strategies Interview (INSI) which measured problem-solving functional behavior in social situations, Behavior Rating Inventory of Executive Function (BRIEF) which measured problem-solving functional behavior in the home environment, and finally the Canadian Occupational Performance Measure (COPM) which measured self-perceived goal-directed functional behavior individually. Up to our knowledge, the outcome measures seem to be dispersed and not fully focusing on a single aspect of cognition as this is obvious in the title of the study as "The effects of problem-solving skills training based on metacognitive principles for children with acquired brain injury attending mainstream schools". It yet showed significance on all levels of outcome measures as reported by the study. Better links should be established between the outcome measures of this study and the functional goals of the training.

\section{Conclusion}

If this review is considered to be valid by the reader, researchers and clinicians should make use of it. On aggregation of Literature level, we were able to detect how MST has been implemented and investigated on different cognitive levels. It might be the interest of other research domains to study whether metacognitive function can influence other impairments. Those impairments are not only cognitive since impression of self plays a vital role in its functionality as reported by Albert Bandura (who was the first to introduce the term "Self-efficacy" that is the personal belief of someone's potential about his own potential). People with higher selfefficacy report higher functionality and wellness of life. Upon the important discussions in neuropsychology, currently, is the macro versus micro understanding of neural networks. One of the causes of this controversy is the development of the instruments used to detect and read neural behavior. Other reasons are the progress of psychotherapy and other rehabilitation techniques on the level of behavior altering and MRI detectable changes on the brain as well the development of understanding conditioning and neural behavior underlying psychiatric and cognitive conditions. This assists us in proposing better framework for our theories and applications when it comes to understanding the brain. If clinicians are to benefit from this study, then it should be acclaimed that MST is not being introduced as a new concept but it is reaffirmed as a protocol for assessment in TBI patients more than simply being an approach in rehabilitation. Yet, the implementation of this approach must be better dissected. Literature has shown that MST is by definition distinct from other approaches which assess and target executive function but metacognitive training sessions and modules are still relative and contrasting and have not yet evolved to be very different from executive function rehabilitation approaches. Executive function has a wide control over other cognitive skills and metacognition structural presence is still controversial. One would say this will allow yielding creativity in the field of explaining executive function and metacognition but it can also be depriving uniqueness required when a protocol is set. Despite MST not being an official protocol, it still stands out to be an essential part of neuro-rehabilitation and cognitive rehabilitation. Metacognitive training can help the patient gain insight and better enhance his error detection skills whether 
they were encompassed in his executive function or not. From a clinical background, metacognitive assessment should be an initial phase added to the rehabilitation in centers equipping TBI patients with skills and generalized daily activity regimes. If it is to give two main recommendations; it is to insert MST in the official protocol of cognitive rehabilitation and other rehabilitation techniques and consider metacognition as an essential component to be assessed before rehabilitation. It is also good to mention that meta-topics are recently being re-explored from new backgrounds. If this review is to offer anything; then it should be "reconsideration" of our official protocols in rehabilitation.

\section{References}

1. Mckee AC, Daneshvar DH (2015) The neuropathology of TBI. Handbook of Clinical Neurology 127: 45-66. Link: https://bit.ly/3jOI1L8

2. Lippi D (2017) Case study of Berengario da Carpi and Lorenzo de' Medici British Journal of Surgery John Wiley and Sons Ltd. 104: 1272. Link: https://bit.ly/3oNhpwm

3. Kabu S, Jaffer H, Petro M, Dudzinski D, Stewart D, et al. (2015) Blast-associated shock waves result in increased brain vascular leakage and elevated ROS levels in a rat model of TBI. Plos One 10. Link: https://bit.ly/324yl7S

4. Jain S, Iverson LM (2020) Glasgow Coma Scale. Link: https://bit.ly/3mBrM4G

5. Rahaman P, Del Bigio MR (2018). Histology of Brain Trauma and Hypoxia-Ischemia. Academic Forensic Pathology 8: 539-554. Link: https://bit.ly/35WCOe8

6. Harvey PD (2019) Domains of cognition and their assessment. Dialogues Clin Neurosci 21: 227-237. Link : https://bit.ly/35PWkJa

7. Arciniegas DB, Held K, Wagner P (2002) Cognitive Impairment Following Traumatic Brain Injury. Curr Treat Options Neurol 4: 43-57. Link: https://bit.ly/2TKgm21

8. Waid-Ebbs JK, Daly J, Wu SS, Berg WK, Bauer RM, et al. (2014) Response to Goal Management Training in Veterans with blast-related mild TBI. Journal of Rehabilitation Research and Development 51: 1555-1566. Link: https://bit.ly/320jNGo

9. Orfei MD, Robinson RG, Bria P, Caltagirone C, Spalletta G (2008) Unawareness of illness in neuropsychiatric disorders: Phenomenological certainty versus etiopathogenic vagueness. Neuroscientist 14: 203-222. Link: https://bit ly/34Nxni2

10. Barman A, Chatterjee A, Bhide R (2016) Cognitive impairment and rehabilitation strategies after TBI. Indian Journal of Psychological Medicine. Medknow Publications 38: 172-181. Link: https://bit.ly/2THIRP2

11. Kennedy MRT, Coelho C, Turkstra L, Ylvisaker M, Sohlberg MM, et al. (2008) Intervention for executive functions after TBI: A systematic review, metaanalysis and clinical recommendations. 18. Link: https://bit.ly/2Jug3X|

12. Finch E, Cornwell P, Copley A, Doig E, Fleming J (2017) Remediation of social communication impairments following TBI using metacognitive strategy intervention: a pilot study. Brain Injury 31: 1830-1839. Link: https://bit.ly/340dDev

13. Dockree PM, O'Connell RG, Robertson IH (2015) Connecting clinical and experimental investigations of awareness in TBI. Handbook of Clinical Neurology 128: 511-524. Link: https://bit.ly/3mK8Sse

14. Ownsworth T, Fleming J, Desbois J, Strong J, Kuipers P (2006) A metacognitive contextual intervention to enhance error awareness and functional outcome following TBI: A single-case experimental design. Journal of the International Neuropsychological Society, 12: 54-63. Link: https://bit.ly/3jJ06ZK
15. Cornis-Pop M, Mashima PA, Roth CR, MacLennan DL, Picon LM, et al. (2012) Cognitive-communication rehabilitation for combat-related mild TBI. Journal of Rehabilitation Research and Development 49: 11-31. Link: https://bit.ly/2TMCA3C

16. Article R, Zettin M, Galetto V (2016) Efficacy of The Metacognitive Training on Brain Injured Subjects. In EC Psychology and Psychiatry 1

17. Dawson DR, Gaya A, Hunt A, Levine B, Lemsky C, et al. (2009) Using the Cognitive Orientation to Occupational Performance (CO-OP) with adults with executive dysfunction following TBI. Canadian Journal of Occupational Therapy 76: 115-127. Link: https://bit.ly/340saXy

18. Franzen KM, Roberts MA, Schmits D, Verduyn W, Manshadi F (1996) Cognitive remediation in pediatric TBI. Child Neuropsychology 2: 176-184. Link: https://bit.ly/361CxXs

19. Kurowski BG, Wade SL, Kirkwood MW, Brown TM, Stancin T, et al. (2014) Longterm benefits of an early online problem-solving intervention for executive dysfunction after TBI in children A randomized clinical trial. JAMA Pediatrics 168: 523-531. Link: https://bit.ly/3jQd48b

20. Ownsworth T, Quinn H, Fleming J, Kendall M, Shum D (2010) Error selfregulation following TBI: A single case study evaluation of MST and behavioural practice interventions. Neuropsychological Rehabilitation 20 59-80. Link: https://bit.ly/3mKavpQ

21. Krasny-Pacini A, Limond J, Evans J, Hiebel J, Bendjelida K, et al. (2014) Contextsensitive Goal Management Training for everyday executive dysfunction in children after severe TBI. Journal of Head Trauma Rehabilitation 29: E49-E64 Link: https://bit.ly/35SG36c

22. Chan DYK, Fong KNK (2011) The effects of problem-solving skills training based on metacognitive principles for children with acquired brain injury attending mainstream schools: A controlled clinical trial. Disability and Rehabilitation 33: 2023-2032. Link: https://bit.ly/35TOVbL

23. Kintz S, Hibbs V, Henderson A, Andrews M, Wright HH (2018) Discourse-based treatment in mild TBI. Journal of Communication Disorders 76: 47-59. Link: https://bit.ly/340t2eM

24. Séguin M, Lahaie A, Matte-Gagné C, Beauchamp MH (2018) Ready! Set? Let's Train!: Feasibility of an intensive attention training program and its beneficial effect after childhood TBI. Annals of Physical and Rehabilitation Medicine 61 : 189-196. Link: https://bit.ly/2TNOMTr

25. Krasny-Pacini A, Limond J, Evans J, Hiebel J, Bendjelida K, et al. (2015) Self-awareness assessment during cognitive rehabilitation in children with acquired brain injury: A feasibility study and proposed model of child anosognosia. Disability and Rehabilitation 37: 2092-2106. Link: https://bit.ly/2Jnu2xX

26. Lebeer J (2016) Significance of the Feuerstein approach in neurocognitive rehabilitation. NeuroRehabilitation 39: 19-35. Link: https://bit.ly/321WQCA

27. Hamzah N, Narayanan V, Ramli N, Veeramuthu V, Tan LK, et al. (2018) A preliminary report on the effect of cognitive rehabilitation therapy in improving cognitive function of attention following mild TBI: A randomised controlled trial. Annals of Physical and Rehabilitation Medicine 61: e41. Link: https://bit.ly/2TJJCWJ

28. Chung CSY, Pollock A, Campbell T, Durward BR, Hagen S (2013) Cognitive rehabilitation for executive dysfunction in adults with stroke or other adult non-progressive acquired brain damage. Cochrane Database of Systematic Reviews 2013. Link: https://bit.ly/3ej1dhC

29. Logan DM, Hill KR, Larson MJ (2015) Cognitive control of conscious error awareness: Error awareness and error positivity $(\mathrm{Pe})$ amplitude in moderate-to-severe TBI TBI. Frontiers in Human Neuroscience 9. Link: https://bit.ly/3ehRrw6

Citation: Makka SA (2020) Metacognitive skills training effect on cognitive function in traumatic brain injury patients: A systematic review. Glob J Medical Clin Case Rep 7(2): 085-099. DOI: https://dx.doi.org/10.17352/2455-5282.000107 
30. McAvinue L, O'Keeffe F, McMackin D, Robertson IH (2005) Impaired sustained attention and error awareness in TBI: Implications for insight. Neuropsychological Rehabilitation 15: 569-587. Link: https://bit.ly/3jQPcRA

31. Levine B, Schweizer TA, O'Connor C, Turner G, Gillingham S, et al. (2011) Rehabilitation of executive functioning in patients with frontal lobe brain damage with goal management training. Frontiers in Human Neuroscience 1-9. Link: https://bit.ly/35Uswv5

32. Perlovsky L, Sakai KL (2014) Language and cognition. Frontiers in Behavioral Neuroscience 8. Link: https://bit.ly/2TKcgXN
33. Genova HM, Haight A, Natsheh JY, DeLuca J, Lengenfelder J (2019) The relationship between social communication and social functioning in pediatric TBI: A pilot study. Frontiers in Neurology 10. Link: https://bit.ly/2HWrTsk

34. TBI (2020) Symptoms and causes - Mayo Clinic. (n.d.). Link: https://mayocl. in/37ZNWcM

35. Kim JJ, Gean AD (2011) Imaging for the Diagnosis and Management of TBI. Neurotherapeutics 8: 39-53. Link: https://bit.ly/3oHDHzE
Discover a bigger Impact and Visibility of your article publication with

\section{Peertechz Publications}

\section{Highlights}

* Signatory publisher of ORCID

* Signatory Publisher of DORA (San Francisco Declaration on Research Assessment)

* Articles archived in worlds' renowned service providers such as Portico, CNKI, AGRIS, TDNet, Base (Bielefeld University Library), CrossRef, Scilit, J-Gate etc.

* Journals indexed in ICMJE, SHERPA/ROMEO, Google Scholar etc.

* OAI-PMH (Open Archives Initiative Protocol for Metadata Harvesting)

* Dedicated Editorial Board for every journal

* Accurate and rapid peer-review process

* Increased citations of published articles through promotions

* Reduced timeline for article publication

Submit your articles and experience a new surge in publication services

(https://www.peertechz.com/submission).

Peertechz journals wishes everlasting success in your every endeavours.

Copyright: () 2020 Makka SA. This is an open-access article distributed under the terms of the Creative Commons Attribution License, which permits unrestricted use, distribution, and reproduction in any medium, provided the original author and source are credited.

Citation: Makka SA (2020) Metacognitive skills training effect on cognitive function in traumatic brain injury patients: A systematic review. Glob J Medical Clin Case Rep 7(2): 085-099. DOI: https://dx.doi.org/10.17352/2455-5282.000107 